\title{
A Review on - Theranostics: An Approach to Targeted Diagnosis and Therapy
}

\author{
Shrivastava Saumya *, Jain Saloni, Kumar Deepak, Soni Shankar Lal, Sharma Mukesh \\ Department of pharmaceutics, Arya College of Pharmacy, Kukas, Jaipur, Rajasthan, India
}

\begin{abstract}
A B S T R A C T
Emerging as a targeted, safe, and efficient pharmacotherapy is the approach of theranostics, which focuses on patient centered care. It is a combination of diagnosis and therapeutics. Theranostics is a new field of medicine which combines specific ttargeted therapy based on specific targeted diagnostic tests. With a key focus on patient centered care, theranostics provides a transition from conventional medicine to contemporary personalized and precision medicine approach. The latest theranostics paradigm involves using nanoscience to unite diagnostic and therapeutic applications to form a single agent, allowing for diagnosis, drug delivery and treatment response monitoring. The theranostics medicine can achieve systemic circulation, evade host defenses and deliver the drug and diagnostic agents at the targeted site to diagnose and treat the disease at cellular and molecular level.
\end{abstract}

Keywords: Diagnosis, therapy, theranostics, nuclear medicine, nanomedicine

A R T I C L E I N F O: Received 23 Dec 2018; Review Completed 25 March 2019; Accepted 09 April 2019; Available online 15 April 2019

Cite this article as:

Shrivastava Saumya *, Jain Saloni, Kumar Deepak, Soni Shankar Lal, Sharma Mukesh, A Review on - Theranostics: An Approach To Targeted Diagnosis And Therapy Asian Journal of Pharmaceutical Research and Development. 2019; 7(2):63-69 DOI: http://dx.doi.org/10.22270/ajprd.v7i2.463

*Address for Correspondence:

Saumya Shrivastava, Arya College of Pharmacy, Kukas, Jaipur, Rajasthan, India

\section{INTRODUCTION}

$\mathrm{T}$ heranostics literally means portmanteau of therapeutics and diagnostics. Theranostics combines therapy and diagnostics to establish more specific and individualized therapies for various pathologies, and to bring about a union of diagnostic and therapeutic applications into a single agent thus leading to a promising therapeutic paradigm involving diagnosis, drug delivery and monitoring of treatment response. ${ }^{1}$ theranostics is a holistic transition from trial and error medicine to predictive, preventive and personalized medicine leading to improved quality care of pharmacotherapy. ${ }^{2}$ It uses specific biological pathways in the human body, to acquire diagnostic images and also to deliver a therapeutic dose of radiation to the patient. A specific diagnostic test shows a particular molecular target on a tumour, allowing a therapy agent to specifically target that receptor on the tumor, rather than more broadly the disease and location it presents. This contemporary form of treatment moves away from the one-medicinefits-all and trial and error medicine approach, to offering the right treatment, for the right patient, at the right time, with the right dose, providing a more targeted, efficient pharmacotherapy in the form of theranostics. The premise behind theranostics is to take advantage of specific biological pathways in the human body to enable the acquisition of diagnostic images. The transformation of these digital images will increase the probability that the targeted therapeutic dose of radiation will reach the disease state and limit the damage to the surrounding healthy tissues. This approach of using a specific diagnostic test identities a particular molecular target on tumor cells to allow a therapeutic agent to bind to the receptor sites and specifically target the regions in the tumor volume.

Mainly for medical purposes: theranostic nanomedicine means colloidal nanoparticles ranging in sizes from 10 to $1000 \mathrm{~nm}(1 \mu \mathrm{m})$. They consist of macromolecular materials/polymers in which the diagnostic and therapeutic agents are adsorbed, conjugated, entrapped, encapsulated for diagnosis and treatment simultaneously at cellular and molecular level. ${ }^{3}$

\section{Conceptuals of Theranostics}

Theranostics helps in the management of various diseases and cancer therapeutics. Cancer therapy greatly relies on conventional radiotherapy and chemotherapy in which anti cancer drugs are taken by the cancer cells at high proliferative rate. The drawbacks of conventional therapy is that the normal cells also get affected by chemotherapeutic action resulting in several side effects.The idea of using a radioactive compound for diagnostic imaging, target-expression confirmation, and 
radionuclide therapy dates back to 1946 , when Seidlin et al. published the first study on radioiodine therapy for metastatic thyroid cancer. ${ }^{4}$ In $19^{\text {th }}$ century, Iodine 131 was identified as first theranostic agent in the treatment of thyrotoxicosis. This isotope when radiated to the patient, it not only identify the benign condition but also helps in the treatment of thyroid cancer. The beta emitting radioactive iodine when administered they selectively kill or harm the thyroid cancer cells and specifically deposit more iodine and the cells can be visualized in gamma camera using gamma rays emitted from Iodine-131. They also helps in identifying the recurrence at an earlier stage depend upon the serum thyroglobulin measurement or radioiodine imaging by removing residual normal thyroid tissues.

Nanomedicine, that is the application of nanotechnology to diagnose, treat and prevent diseases at cellular and molecular level, can also be efficient for development of theranostic candidate to combine diagnosis and therapy simultaneously. An aadvanced theranostic nanomedicine is multifunctional in nature, capable of diagnosis and delivery of therapy to the diseased cells with the help of targeting ligand and biomarkers. They consist of macromolecular materials/polymers in which the diagnostic and therapeutic agents are adsorbed, conjugated, entrapped, encapsulated for diagnosis and treatment simultaneously at cellular and molecular level.eranostic nanomedicine can work better than other theranostics since they have advanced capabilities in an all-in-one single platform, which include sustained/controlled release, targeted delivery, higher transport efficiency by endocytosis, stimulus responsive agent release (i.e., smart delivery), synergetic performance (e.g., combination therapy, siRNA co-delivery), multimodality diagnosis and/or therapies and quality performances (e.g., oral delivery, escape from multi drug resistance (MDR) protein, autophagy inhibition etc).The encapsulation of single diagnostic or therapeutic agent in nanomedicine may not have high efficacy/specificity/sensitivity for the applications. Therefore, multimodality theranostics can be developed to make use of the advantages, which may be achieved by co encapsulation of multiple diagnostic modes and therapeutic modes in targeting platforms.

A variety of platforms are exploited for the theranostic purposes, which are summarized as below-

- Radioisotopes

- Genetic materials (DNA sequences, RNA and proteins)

- Quantum dots

- Dendrimers

- Liposomes

- Magnetic Nanoparticles

- Fullerenes and Nanotubes

- Gold Nanoparticles

- Microbubbles

- Porous nanoparticles

- Micelles

- Drug-polymer conjugates

- Antibodies

THERAPEUTIC NUCLEARMEDICINE: "THE GOLD STANDARD” OF THERANOSTICS

The first successful theranostic radiopharmaceutical in nuclear medicine history was radioiodine, which was used for therapy and imaging in thyroid diseases. Since then, the use of theranostic agents has been consistently increasing. Nuclear targeted therapies play an essential role, especially in patients with advanced neuroendocrine tumors, such as gastroentero-pancreatic (GEP) tumors, broncho pulmonary neuroendocrine tumors, pheochromocytoma, and neuroblastoma. Furthermore, there are positive results with radioligand therapies in metastatic prostate cancer and metastatic melanoma. ${ }^{5}$

The theranostic approach in nuclear medicine couples diagnostic imaging and therapy using the same molecule or at least very similar molecules, which are either radio labeled differently or given in different dosages. For example, iodine-131 and lutetium-177 are gamma and beta emitters; thus, these agents can be used for both imaging and therapy. Furthermore, different isotopes of the same element, such as, iodine-123 (gamma emitter) and iodine-131 (gamma and beta emitters), can also be used for theranostic purposes. Newer examples are yttrium-86/yttrium-90 or terbium isotopes (Tb): $152 \mathrm{~Tb}$ (beta plus emitter), 155Tb (gamma emitter), 149Tb (alpha emitter), and $161 \mathrm{~Tb}$ (beta minus particle). ${ }^{6}$

The nuclear theranostics approach is gaining pace through a series of milestones including:

- Lutetium PSMA therapy for metastatic or treatmentresistant prostate cancer

- Yttrium-90 SIRT therapy for liver cancer

- Iodine-131 therapy for thyrotoxicosis and thyroid cancer

- Radium-223 therapy for metastatic prostate cancer in bones

- Yttrium-90 radiosynovectomy therapy for inflammatory synovitis of joints.

The detection of potential targets can help predict whether a patient will benefit from a particular treatment. Theranostics can be useful for estimating the potential response and eventual toxicity. During the treatment, theranostics can be applied in monitoring the therapy course. However, one cause of concern is the safety of high cumulative doses of radioactive agents after multiple repeated cycles.

Iodine (stable isotope: iodine-127) is taken up by the thyroid gland for the production of thyroid hormones, namely, thyroxin (T4) and tri iodothyronine (T3). Thyroid hormones are vital for the embryonic and neonatal development of the brain, normal growth, and metabolic balance. In 1936, Dr Saul Hertz, director of the Thyroid Clinic in Massachusetts, developed the idea of administering radioactive iodine in patients with thyroid diseases. Iodine and external beam radiation were wellknown tools in thyroid disease therapy, but the combination of these was a considerably innovative approach.

This idea followed a few years of preclinical studies in collaboration with the Massachusetts Institute of Technology (MIT), where the first cyclotron for medical use had been built. The MIT Cyclotron produced 90\% iodine-130 (130I, half-life: 12 hours) and 10\% iodine-131 (131I, half time: 8 days). Subsequently, on March 31, 1941, Dr Hertz treated the first patient with radioiodine (130I).The first radioiodine therapy (RAI) with 131I in 
patients with thyroid cancer (TC) was undertaken by Seidlin et al in $1946 .^{5}$

To date, 131I is still the gold standard for the therapy and diagnosis of differentiated TC.38 It is a low-cost nuclear reactor product from the neutron bombardment of tellurium-131. 131I combines the characteristics of a beta ( $\beta^{-}$, approxi-mately $90 \%$ of the radiation, mean: $192 \mathrm{keV}$, mean tissue penetration: $0.4 \mathrm{~mm}$ ) and gamma (approximately $10 \%$ of the radiation, mean: $383 \mathrm{keV}$ ) emitter. In this way, it irradiates the TC and the thyroid remnant from the inside and, at the same time, targeted lesions can be visualized using a gamma camera or SPECT.9,32,33 Another radioisotope is 123I, which is a pure gamma emitter and is used for pre- and posttherapeutic diagnostics. ${ }^{7}$

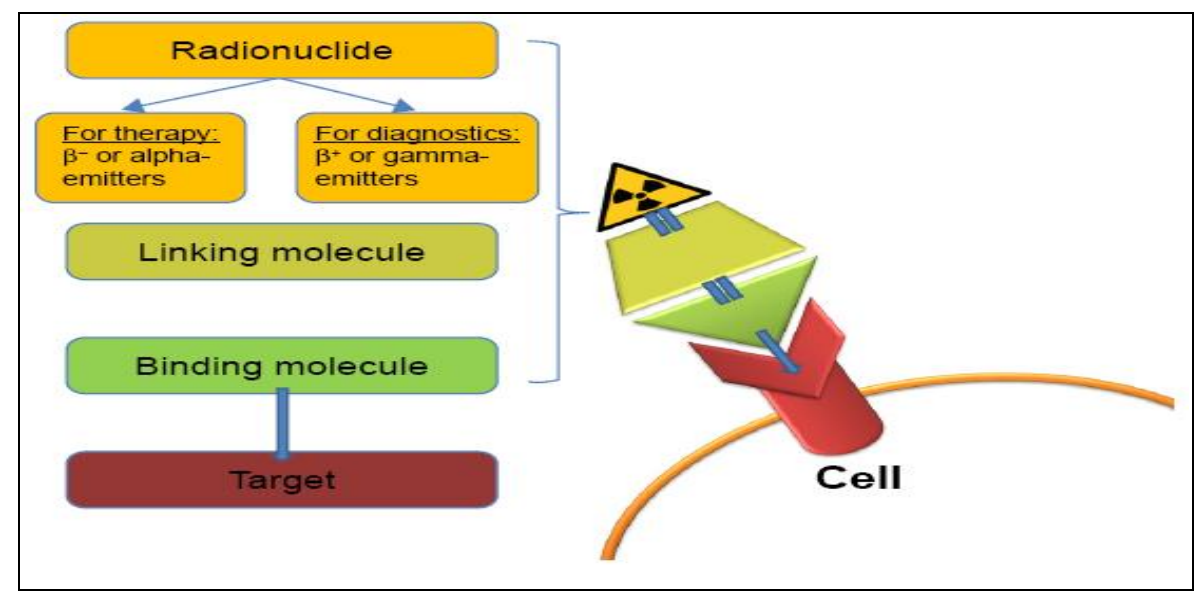

Figure 1: Representation for action of nuclear theranostic agent

The image shows a simplified model of a radiopharmaceutical, which consists of a binding molecule that binds the target, and a linking molecule, which binds the radioisotope. The theranostic principle in nuclear medicine involves combining diagnostic imaging and therapy with the same molecule, which is radio labeled differently, or administered in other dosages. In case of radioiodine therapy (RAI), the radioisotope (131Ior 123I) can be directly mediated by the sodiumiodide symporter in the thyroid cells. In other cases, it can be more complex.

\section{THE ADVANCED AGENTS - NANOTHERANOSTICS}

Theranostic nanomedicine may be defined as nanomedicine that combines diagnostics with therapeutics. Indeed, some nano medicines have intrinsic diagnostic as well therapeutic properties. They act as self theranostic nanomedicines or platforms. The advanced theranostics nanomedicine conjugated with targeting moiety can recognize specific target, bind and be internalized via specific mechanism e.g., receptor mediated endocytosis.
Nanomedicine offers multiple and desirable advantages over conventional drug products. First, the nanocarrier scan be easily prepared using standard procedures in nanotechnology to provide designed functionalities and to achieve specific targeting. Second, nanocarriers are competent enough to improve the pharmacokinetics and to enhance the biodistribution of existing therapeutic moieties to the target tissues, so that can improve efficiency. ${ }^{8}$

Third, nanocarriers have a promising intrinsic advantage like site specific delivery within solid tumors via the leaky vasculature. Nanocarriers can further improve the safety index of the anticancer agent by using non-toxic or biocompatible polymers which inhibit the release of therapeutic agent within normal tissues and thus, reducing overall systemic toxicity. Fourth, most of the nano systems possess the inherent advantage of enhancing aqueous solubility of lipophilic compounds and make them appropriate for administration through parenteral route. Fifth, the loading or encapsulation of therapeutic entities such as small sized hydrophobic molecules, peptide drugs as well as oligonucleotides within the nanosystems enhance their stability as well. ${ }^{8}$

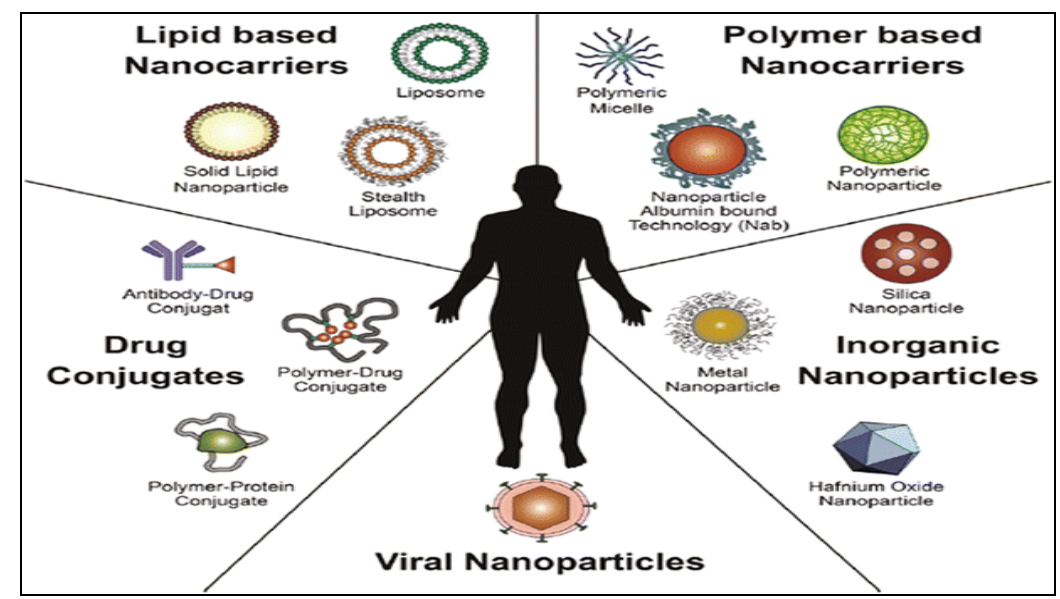

Figure 2: Various types of nanotheranostics 
Due to its size, nanomedicine is able to move freely through small blood capillaries and gain access to cancer cells in the target tissue. In addition, if a diagnostic molecule is attached, it is also possible to sense the level of curative effect within the brain tumor. As a matter of fact, novel therapeutic and diagnostic principles, advanced diagnostics and novel targeted approaches can be unified and prosper with the aid of nanotechnology. ${ }^{9}$

Pharmacogenomics - Pharmacogenomics extends to involve variations in RNA and proteins also that may have important role in treatment. Genetics play an important role in theranostics and promoted by human genome project to identify the variation in genes. The components of nanoparticles includes diagnostic agent, Therapeutic agent, Targeting agent and Carrier.

Quantum Dots - Quantum dots are the nano crystals discovered by Dr. Bawendi. They have advanced the field of diagnostics and nano therapeutics by their high surface volume ratio and act as a nano scaffold for theranostic modalities.

Dendrimers - Dendrimers are macromolecular structures that consists of a series of branches around an inner core that can encapsulate therapeutic agents within their core and also incorporated with cell identification tags, fluorescent dyes, enzymes and other molecules onto the "hooks" present on their surface. They can be used in the different field of theranostics like chemotherapeutic theranostics, biotherapeutic theranostics, phototherapeutic theranostic, radio therapeutic theranostics and combined therapeutic theranostics.

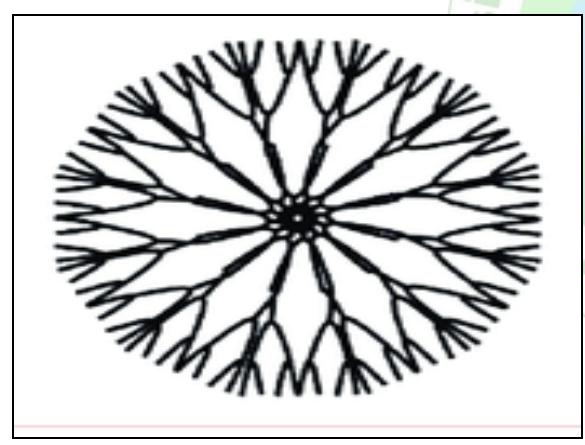

Figure 3: Dendrimer

Liposomes - Liposomes invented by Alec D Bangham at the Babraham Institute in the University of Cambridge in 1960. It is made up of single or multiple concentric lipid bilayers incorporating an aqueous compartment. The liposome-based formulations for gene therapy, such as synthetic cationic liposomal-DNA called lipoplexes, have greater potential to utilize in oral cancer treatment.

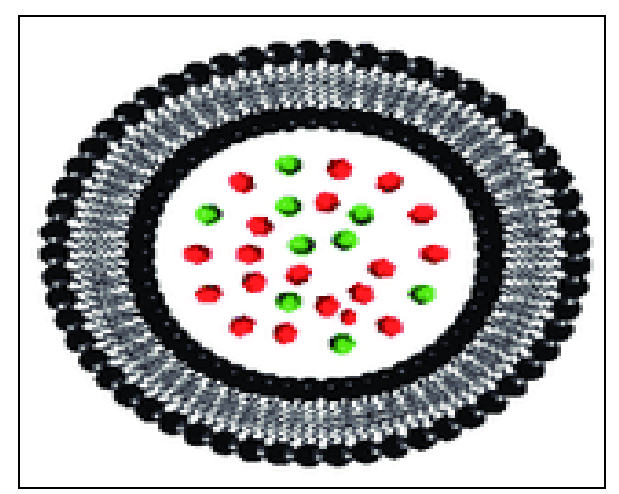

Figure 4: Liposome
Micelles - Micelles are self-assembling colloidal structure with a hydrophobic core and hydrophilic shell. They have narrow size distribution and less than $100 \mathrm{~nm}$ in size. Therapeutic/diagnostic agents can be loaded into hydrophobic core of micelles and theouter hydrophilic layer with targeting agent, which can then be administered intravenously. The theranostic micelles of particle size less than $50 \mathrm{~nm}$ in diameter avoid renal exclusion, reticulo endothelial system and provide them with enhanced endothelial cell permeability in solid tumors.

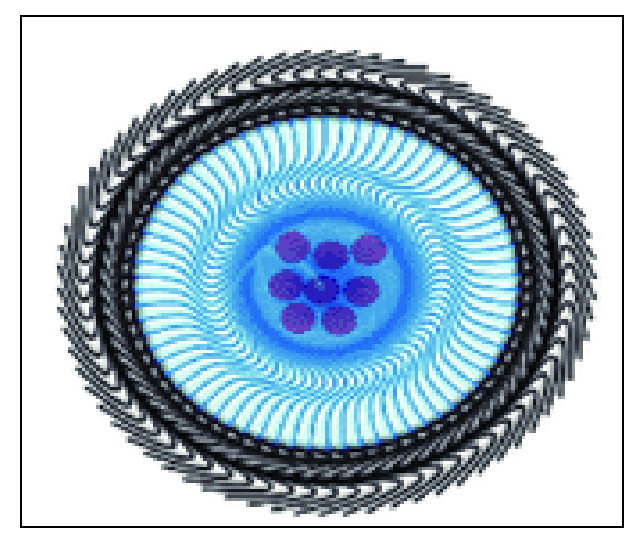

Figure 5: Polymeric micelle

Magnetic Nanoparticles - Magnetic nanoparticles are widely used for nano particle based drug delivery systems. They can be made in different sizes and can be surface coated in order to carry the molecules. Nanoparticles are used in transporting various substances like antimicrobial molecules, genes, proteins and anti cancer drugs.

Fullerenes and Nanotubes - Fullerenes are also known as "bucky balls" available in the shape of hollow or ellipsoid pattern. Fullerenes have the potential to induce the host immune response and formation of fullerene specific antibodies. Nanotubes are the tube like structures available as carbon nanotube, inorganic nanotube, DNA nanotube, membrane nanotube etc. They also used to transport DNA across cell membrane during gene therapy.

Gold Nanoparticles - Gold nanoparticles (AuNPs) have been used as drug carriers, photo thermal converters, radiosensitizers, and imaging probes in cancer diagnosis and therapy. AuNPs are distinguished into nanospheres, nanorods, nanoshells, nanocages, and surface- enhanced Raman scattering (SERS) nanoparticles, based on their size, shape, and physicochemical properties. AuNPs have many features that make them unique in cancer theranostics.

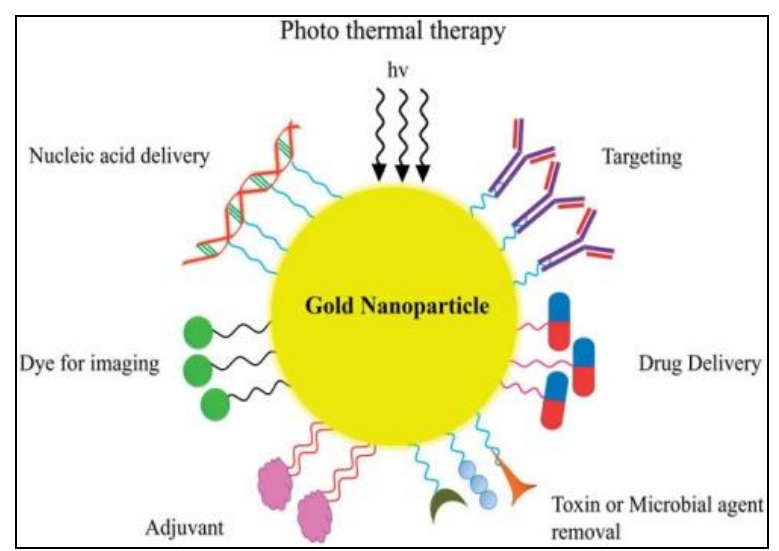

Figure 6: Gold nanoparticle 
Microbubbles as Cancer Theranostics - Microbubbles are smaller in size and used in various fields of science. Conventional micro bubbles of micron diameter cannot escape from the blood pool, the initial target is the blood vasculature. It helps to identify the tumour neovascularization. They temporarily open the blood vessels and conduct the impulses for the endothelial barrier for a while and helps in therapeutic and diagnostic purposes.

Antibodies-To date, several mAbs have been approved for thetreatment of cancer. Antibodies specifically target antigens expressed on the cell membrane or ligands and can either be administered un conjugated or conjugated to cytotoxic moieties such as radionuclides or toxins to increase efficacy. ${ }^{10}$

\section{Components of Nanoparticle Based Drug Delivery System}

Theranostics composed of carrier agents like liposomes, dendrimers, and polymeric nanoparticles, carbon nanotubes incorporated with plasmid, nucleic acids and proteins helps to diagnose with the help of quantum dots, gold nanoshells to target the peptides, antibodies, vitamins. The nanoparticles are conjugated into the drug payload containing a Y-shaped ligand deliver the diagnostic and therapeutic agent into the surface of tumor cells. They undergo enhanced permeability and retention. The tumor cell also develops its vasculature than the normal cells thereby allowing the drug to pass through the interstitial space. $^{11}$

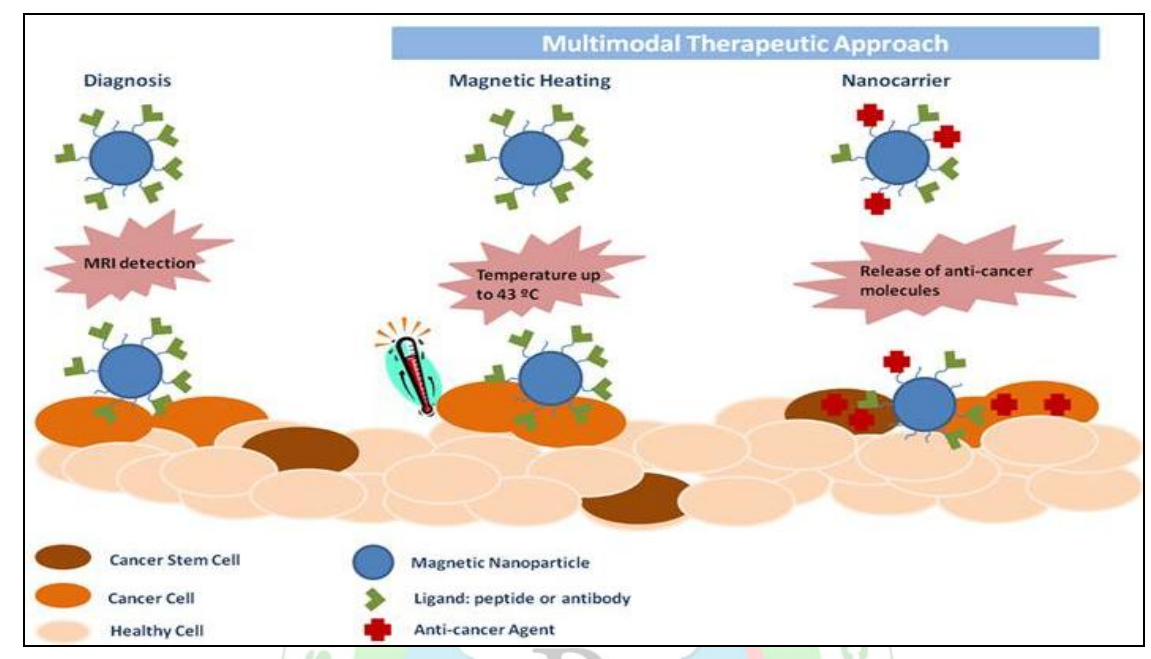

Figure 7: Activity of Theranostics: MRI detection + multimodal therapeutic approach

\section{CHALLENGES IN THERANOSTICS}

The fundamental principle of theranostics has been followed since decades, with the utilization of radioiodine todiagnose (image) and manage cancers of thyroid gland which dates back to 1940 . Since its inception until date, theranostics has been fostering unremarkable tailored and targeted therapy. ${ }^{12}$

In the context of pharmacotherapy of diseases like cardiovascular diseases and AIDS, the fundamental aspectof individual absorption distribution metabolism excretion is to understood with regards to the complex interactions and detailed characterization, especially focusing on the functional variations genetically also including population variations.

Furthermore interestingly, epigenetic variations and the impact of environmental factors including the circadian rhythms on an individual's response to drugs needs to be assessed specific to each individual. Apart from the above variations educating the health care provider, payor, regulator, and the patient also is mandatory.

Theranostics has extended impact on patients in that more than individual centric care, guaranteed outcomes expected by patients seem to pose an alarming aspect of theranostics. Due to existing less targeted and tailored pharmacotherapy, the resulting loss in $40 \%$ of ineffective treatment has resulted in loss in overall economy of the pharmacological market accounting to $\$ 400$ billion out of total global pharmaceutical market $\$ 825$ billion. Our country accounts to $20 \%$ of global population and involves $2 \%$ of global pharmaceutical market, especially with respect to generic drugs. Every year after conducting clinical trials, approval by the Central Drugs Standard Control Organization has considerably decreased since 2009. The most common reason for failure and avoidable deaths, including expensive hospitalization has been found to be side effects associated with less targeted nonspecific therapy contrary to theranostics based management. Furthermore age, and gender, diet, lifestyle of a patient including the intestinal micro flora playsa significant role in patient's response to a drug. ${ }^{12}$

The development of molecular diagnostic tests and targeted therapeutics in an interdependent and collaborative manner, particularly focusing on individualizing treatment by targeting therapy to an individual's specific disease subtype and genetic profile, enables optimization of drug efficacy and safety, assisting in streamlining the drug development process. The companion diagnostic is a tool that helps in determining the subtype of the disease, its progression, and the characteristics of a patient. $^{12}$

\section{APPLICATION OF THERANOSTICS IN PATHOLOGY}

Today's Biomarker Tomorrows Theranostics Theranostics helps in the identification of biomarker that help the medical practitioners to treat the individual patients depend upon their biomarker profile thereby enhancing the bioavailability and reducing the side effects. The ongoing research going on in this particular field to develop this field of drug development. 
Chemotherapy - The one of the most common drug used for the conventional treatment of cancer is cisplatin. The various researches proves that hydrophilic poly (ethylene glycol) shell layer enables the particles to circulate for long time in the blood compartment which will facilitate its preferential accumulation in the tumor tissues. Curcumin NPs (Cur-NPs) induce cell apoptosis in CAL27- cisplatin resistance human oral cancer cells (CAR cells) and inhibit cell growth but possess little cytotoxicity to normal human gingival fibroblasts cells and normal human oral keratinocyte cells. The findings suggest that Cur-NPs trigger apoptotic cell death through regulating the function of MDR1 and the production of reactive oxygen species, and the activation of caspase- 9 and caspase - 3 connected to intrinsic signaling pathway is the major pharmacologic action of Cur-NPs. Cur-NPs show promise for development as a novel medicine against cisplatin resistant human oral cancer.

Biotherapy - The gene therapy is method of transferring genetic materials into specific cells to treat diseases. These genes act therapeutically by regulating the amount of proteins, adjust the gene expression and generate cytotoxic proteins. Non viral gene vectors helps in efficient loading of genes in the region but they have certain limitations like they remain unstable when circulate in the blood stream and cell specific targeting system needed for the effective process. So drug delivery can be mediated by nanoparticle based theranostic reagents.

Phototherapy - Nanoparticles help in delivering the photo sensitizers to the site of lesion and induce cell apoptosis. They also promote excitation and auto-fluorescence to endow with theranostic modality.

Photo Thermal Therapy - Photodynamic therapy (PDT) achieves its therapeutic effects through the different mechanism from drug and gene delivery. It also helps in delivering an anticancer drug or protein or altering gene expression, nanotheranostic particles used for this purpose destroy their targets. This is a minimally invasive technique that kills target cancer cells in the presence of oxygen by the release of reactive oxygen species upon light activation of a photosensitizer. This kills the cancer cells through direct cellular damage, vascular shutdown, and induction of the host immune response to oppose the target cells.

Cytology Based Theranostics in Oral Squamous Cell Carcinoma - It was found in the study that theranostics along with oral brush cytology proved to the best way in profiling the biomarker in oral squamous cell carcinoma diagnosis. The prognostic significance of the epidermal growth factor receptor (EGFR) family and the angiogenic pathway in oral squamous cell carcinoma are also correlated with help of the targeted therapy.

Immune Response -Nanoparticles containing drug molecules called interleukins are attached to certain immune cells (T-cells). Nanoparticles will release the Tcells along with the drugs and the $\mathrm{T}$ cells are reproduced in it. It results in destroying the cancer cells. Combining the effects of magnetic nanoparticles that attach to cancer cells in the blood stream may allow the cancer cells to be removed before they establish new tumours.

Cancer Therapy -Molecular diagnostics by imaging used to locate the tumour to guide target-specific therapy. Monoclonal antibodies reduce the systemic toxicity and provide the synergistic effect with the nanomedicine formulation. Nanoparticles which emit light of different colours could helps in the identification of different markers. Nanoparticles can be developed in the way that is naturally biodegradable and they can be eliminated easily from the body by natural metabolism.

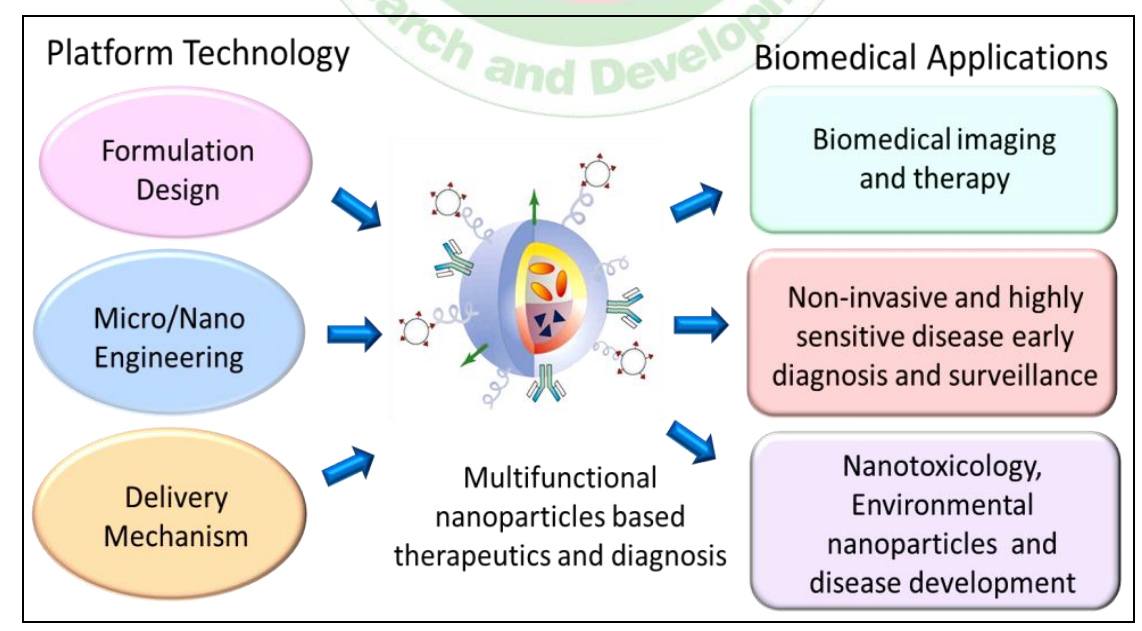

Figure 8: Theranostics application

\section{FUTURE PERSPECTIVE}

The future dynamics of the field of theranostics are anticipated by introducing new radioisotopes and describing individualized nanomedicine.

Major aspects in cancer nanotechnology include early cancer diagnostics, tracking tumor metastasis, imaging tumor angiogenesis, tracking intracellular delivery of therapeutic drugs and monitoring tumor therapy. Multifunctional theranostic agents could certainly have tremendous potential to address some of these pressing challenges in the near future. ${ }^{13}$

In nuclear medicine, theranostics combine diagnostic imaging and therapy with the same, but differently radiolabeled, molecule, or the same agent in different dosages. The visualization of potential targets can help predict if a patient would benefit from a particular treatment. In properly preselected patients, targeted nuclear therapies have proven to be effective with a favorable safety profile. To conclude, the combination of targeted cancer imaging 
and therapy is a considerable contribution to personalized medicine and may play an increasingly important role in the future.

Recent research has shown that well-designed nanosystems could communicate in vivo to amplify the cancer-targeting efficacy.There is also a growing interest in developing activatable theranostic nanoparticles for even "smarter" cancer imaging and therapy. ${ }^{14}$

A seamless and interesting integration of multiple imaging and treatment technologies within a single nano particle is required to tackle pathologies. Nanorobots approximately

\section{REFERENCES}

1. Thangadurai M, Nalliapan G, Janardhanam DS, et al. Theranostics an Emerging Paradigm- a Review. IOSR Journal of Dental and Medical Sciences. 2018; 17(11): 01-07.

2. Jeelani S, Reddy RC, Maheswaran T, Asokan GS, Dany A, Anand B. Theranostics: A treasured tailor for tomorrow, J Pharm Bioallied Sci. $2014 ; 6(1)$ :S6-8.

3. Madaswamy S. Muthu, David Tai Leong, Lin Mei, Si-Shen Feng. Nanotheranostics - Application and Further Development ofNanomedicine Strategies for Advanced Theranostics, Theranostics 2014; 4(6):660-677.

4. Ken Herrmann, Steven M. Larson and Wolfgang A. Weber. TheranosticConcepts: More Than Justa Fashion TrendIntroduction andOverview. J Nucl Med, 2017; 58(2).

5. Anna Y, Elisabeth E, Stefan K, Ralph AB, Stefan S, Maria GC, Georg F, Hojjat A, Markus E. Theranostics in nuclear medicine practice. OncoTargets and Therapy: Dovepress, 2017;10

6. Warland S, Hesse M, Renauld L, Jumar F. The impact of image reconstruction bias on $\mathrm{PET} / \mathrm{CT}^{90} \mathrm{Y}$ dosimetry after radioemobilisation. J Nucl Med, 2015; 56(3):494-495.

7. LusterM, Clarke S.E, Dietlein M, Lassmann M, et al. Guidelines for radioiodine therapy of differentiated thyroid cancer. Eur J Nucl Med Mol Imaging. 2008; 35(10):1941-1949.

8. Sonali, Viswanadh MK, Singh RP, Agrawal P, Mehata AK, Pawde DM, Narendra , Sonkar R, Muthu MS. Nanotheranostics: Emerging the dimension of bacteria probably 1 day may roam human's bodies tracking out organisms and repairing damaged body tissues. ${ }^{15}$

Thus, theranostics approach can be considered as "P4 medicine, "that is predictive, preventive, personalized, and participatory. This field promises to increase the quality of clinical care and treatments and will ultimately save costs thus helps to identify the right drug for the right patient at the right time. Implementation of appropriate theranostics can significantly improve efficacy, efficiency and cost effectiveness of modern treatments. ${ }^{16}$

Strategies for Early Diagnosis and Therapy of Brain Cancer. Nanotheranostics 2018; 2(1):70-86.

9. Shapiro A, Livney YD, broxterman HJ, Assaraf YG. Nanomedicine for Targeted Cancer Therapy: towards the overcoming of drug resistance. Drug Resist Update 2011; 14(3):150-163.

10. mmy D.G. Fleuren, Yvonne M.H., Versleijen-Jonkers, Sandra Heskamp, Carla M.L. van Herpen,Wim J.G. Oyen, Winette T.A. van der Graaf, and Otto C. Boerman. Theranostic applications of antibodies in oncology. Mol Oncol. 2014; 8(4): 799-812.

11. Hegdekar CN. Nanotechnology and the theranostic approach for the future management of cancer and the ethics of nanomedicine. 2010, medlink-uk.net.

12. Nimita L. Pharmacogenomics, Theranostics and Personalised medicine- The complexities of clinical trials: challenges in the developing world. Appl Transl Genomics, 2013; 2:17-21.

13. Santra S. The potential clinical impact of quantum dots. Nanomedicine (Lond). 2012; 7(5):623-626.

14. Feng Chen, Emily B. Ehlerding and Weibo Cai. Theranostic Nanoparticles. J Nucl Med. 2014 Dec; 55(12): 1919-1922.

15. Bhattarai N, Bhattarai SR. Therapeutic nanoparticles: A recent breakthrough in Nanotechnology. J Nanomed Nanotechnol. 2012; 3:e114.

16. Sairamesh J, Michael R. An economic perspective on personalized medicine. Hugo J. 2013; 7:1. 